\title{
Task-Contingent Persistence is Related to Better Performance-Based Measures in Patients with Chronic Musculoskeletal Pain
}

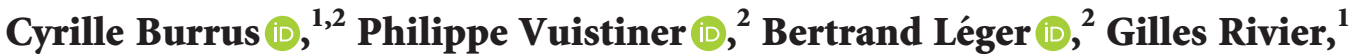 \\ Roger Hilfiker, ${ }^{2,3}$ and François Luthi ${ }^{1,2,4}$ \\ ${ }^{1}$ Department for Musculoskeletal Rehabilitation, Clinique Romande de Réadaptation Suva, Avenue Grand-Champsec 90, \\ 1950 Sion, Switzerland \\ ${ }^{2}$ Institute for Research in Rehabilitation, Clinique Romande de Réadaptation Suva, Avenue Grand-Champsec 90, \\ 1950 Sion, Switzerland \\ ${ }^{3}$ School of Health Science, University of Applied Sciences and Arts Western Switzerland Valais (HES-SO Valais-Wallis), \\ 1950 Sion, Switzerland \\ ${ }^{4}$ Department of Physical Medicine and Rehabilitation, Orthopaedic Hospital, Lausanne University Hospital, Rue du Bugnon 21, \\ 1011 Lausanne, Switzerland
}

Correspondence should be addressed to Cyrille Burrus; cyrille.burrus@crr-suva.ch

Received 3 October 2019; Accepted 20 May 2020; Published 17 June 2020

Academic Editor: Fabio Antonaci

Copyright ( 92020 Cyrille Burrus et al. This is an open access article distributed under the Creative Commons Attribution License, which permits unrestricted use, distribution, and reproduction in any medium, provided the original work is properly cited.

Purpose. Pacing, avoidance, and overdoing are considered the three main behavioral strategies, also labeled activity patterns. Their relationship with functioning of patients with chronic pain is debated. The purpose of this study was to measure the influence of activity patterns on lifting tasks commonly used in daily life. Method. We performed a monocentric observational study and included patients performing Functional Capacity Evaluation (FCE). Avoidance, pacing, and persistence were assessed with using the Patterns of Activity Measures-Pain (POAM-P). Maximal safe performance was measured for floor-to-waist, waist-tooverhead, horizontal lift, and carrying with dominant-hand tests according to the FCE guidelines. Descriptive statistics, associations of POAM-P subscales with various sociodemographic variables, and correlations are presented. Standard multiple linear regression models were applied to measure the associations between FCE tests and POAM-P subscales, adjusting for the following potential confounders: age, gender, body mass index (BMI), pain severity, trauma severity, localization of injury, and education. Results. Persistence was significantly positively associated with performance on the 4 FCE tests: floor-to-waist (coefficient $=0.20 ; p=0.001)$, waist-to-overhead (coefficient $=0.13 ; p=0.004)$, horizontal lift $($ coefficient $=0.31 ; p \leq 0.001)$, and dominant-handed lifting (coefficient $=0.19 ; p=0.001$ ). Pacing was found to have a negative influence on the carrying dominanthand test (coefficient $=-0.14 ; p=0.034$ ), and avoidance was not found to have an influence on the 4 FCE tests. Conclusion. This study shows that task-persistence pattern is positively associated with physical performance in FCE, whereas pacing can have a negative influence on some tests.

\section{Introduction}

The influence of behavior on the development and perpetuation of chronic pain has been the subject of debate for many years $[1,2]$. There are generally 3 main behavioral strategies considered [3] also labeled activity patterns, which can be used to varying degrees and combinations by patients with chronic pain, depending on the context [4]: avoidance (defined as a decrease or escape of physical activity due to fear of movement or fear of pain), pacing (described as a regulation using alternating periods of activity and rest often with the objective of controlling pain or fatigue), and persistence (described as continuing activity despite pain with the risk of detrimental effects). Persistence, in contrast to 
other patterns, has been given various designations: endurance, overactivity, persistence, confrontation, or overdoing in the original Cane study [5]. The construct of the overdoing pattern measured with the Patterns of Activity Measure- Pain (POAM-P) used in this study has been shown by Kindermans to reflect a task-contingent persistence pattern [6]. To be in agreement with both Cane's choice and the underlying construct, this pattern will be labeled overdoing/persistence in this study. The relationship between these patterns and the functioning of patients with chronic pain remains subject to significant controversy [7-9]. One of the reasons for this may be the discrepancies often observed between subjective measures of functioning and observational measures [10, 11]. For instance, disability questionnaires are consistently associated with the intensity and duration of pain, while observational measures of daily living activities often show little or no difference $[6,12]$. Improving physical capacities is an important therapeutic objective of rehabilitation. In particular, knowledge of one's own ability to carry a load safely has multiple implications for daily life and social participation of people with chronic pain: for example, shopping, carrying a child, or performing different physical tasks in many jobs. In clinical conditions, it is sometimes challenging to transfer standardized and controlled measurement conditions that are used in the laboratory [13]. Consequently, the level of maximal safe physical performance achieved by patients with chronic pain may be particularly hard to determine [14]. Functional Capacity Evaluation (FCE), frequently employed in rehabilitation to determine physical performance, is one of the ways to overcome this discrepancy [15]. It is based on standardized measurements, with a clear definition of a safe maximal performance, and has been validated [15]. It has good to excellent reliability [16-18]. Self-efficacy and various biological factors such as age, body mass index (BMI), and gender are known to influence FCE performance $[15,19-21]$. The relationship between psychological variables and FCE is debated $[15,21]$. To date, no studies have evaluated the possible associations between behavioral activity patterns and FCE in patients with chronic musculoskeletal pain. In a previous study, however, we found that the overdoing/persistence pattern was positively associated with commonly used performance-based measures (walking, lifting (pile-test), and physical fitness). No consistent association was found with the avoidance pattern, and pacing was negatively associated with walking performance [22]. In another setting, pacing activity pattern was associated with lower levels of perceived disability [3]. There is a need to better understand behavioral factors influencing physical activity and the barriers that could explain the lack of improvement of physical performances, despite the bestdesigned rehabilitation program [23].

The aim of our study was to investigate the influence of activity patterns on patients with chronic musculoskeletal pain during FCE by using the POAM-P questionnaire and to answer the following question. What are the associations between the 3 activity patterns and the FCE lifting performances in patients with chronic musculoskeletal pain? Based on previous research addressing observational measurements [22], our hypothesis was that overdoing/ persistence would be positively associated with FCE, whereas pacing and avoidance would not show consistent associations with FCE.

\section{Materials and Methods}

2.1. Study Design. We conducted an observational study at the Clinique Romande de Réadaptation, a rehabilitation center in the French-speaking part of Switzerland.

2.2. Participants. Any patient of working age (18-65 years old) who had an FCE from October 2013 to April 2018 was eligible. As previously described [15], exclusion criteria were patients with spinal cord or traumatic brain injuries, incapable of judgment or under legal custody, and with inability to complete the questionnaire in French. The setting of rehabilitation $[15,22]$ of patients consisted of patients from French-speaking urban or more rural regions of Switzerland are referred from by general practitioners, surgeons, or insurance medical advisors when they present with chronic musculoskeletal pain ( $\geq 3$ months), functional impairments, and inability to return to work after orthopedic injuries following work, traffic, sport, or leisure activities [15, 22]. Patients undergo outpatient rehabilitation close to their residence and are sent to a specialized rehabilitation center in case of unfavorable evolution or they are unable to resume their professional activity. The aim of the therapeutic program is to manage patients using a multidisciplinary biopsychosocial approach according to the practice recommendations for chronic pain patients [24]. Individual and group sessions are offered including physical and occupational therapies. Therapies are based on graded and functional trainings, with trainings in professional training workshops, social advice, and psychological components with an average of 4 psychological cognitive-behavioral therapy periods. The mean length of stay is between four and five weeks, with around four hours of therapies every working day. The inclusion period ran from October 2013 to April 2018. If a patient was treated twice during this period, we considered data from only the first rehabilitation.

All participants read and signed an informed consent form to allow access and the use of their clinical data for the present study. The protocol was approved by the ethical committee of the local medical association (Commission Cantonale Valaisanne d'Ethique Médicale, CCVEM 034/12), and the study was performed in accordance with the ethical standards listed in the 2008 Declaration of Helsinki.

2.3. Participants'Data. Patients' sociodemographic data and variables of interest were chosen for their possible confounding influence on the FCE performances [21]. They were collected at the time of admission by the doctor in charge and included (1) age, (2) gender, (3) body mass index (BMI), (4) injury location (upper limb, lower limb, spinal, or multiple-site injuries), (5) trauma severity based on the Abbreviated Injury Scale score (minor injury versus moderate or serious injury) [25], (6) duration from injury to 
rehabilitation (days), (7) first language (French versus others), (8) education level ( $\geq 9$ years of schooling: yes versus no), (9) employment contract (yes versus no), and (10) work-related injury (yes versus no).

2.4. Questionnaires. The Hospital Anxiety and Depression Scale (HADS) [26] was used to assess depression and anxiety symptoms. The HADS consists of 2 subscales, each containing 7 questions (range, 0-21), with a higher score suggesting higher depressive or anxious symptoms [27]. We used the validated French version [28].

For pain assessment, we used 2 subscales of the Brief Pain Inventory (BPI) questionnaire. The severity subscale measured pain at its "worst," "least," "average," and "now" state with 4 numeric scales and was scored as the mean (scale, $0-10$ ), with a high score indicating high severity [29-31]. The interference subscale measured how much pain interferes with seven daily activities. It was scored as the mean of the seven interference items (scale, $0-10$ ), with a high score indicating a high pain-related disability [29-31].

Perceived functional ability was measured by the Hand Function Sort (HFS) and the Spinal Function Sort (SFS), which are pictorial questionnaires assessing the perceived capacity to achieve physical activities related to daily and work activities, including material handling, postural tolerance, and ambulation [15]. The HFS (62 tasks; range, $0-248$ ) was used for patients with upper limb injuries $[32,33]$ and the SFS (50 tasks; range, $0-200$ ) for patients with spinal or lower limb injuries $[34,35]$. To be able to compare the scores, we rescaled the HFS score to a maximum of 200 points. The higher the score, the higher the perceived functional ability.

\subsection{Contributing Factors}

2.5.1. POAM-P. Avoidance, pacing, and overdoing/persistence were assessed at entry using POAM-P, developed by Cane [5] and validated in French [36]. The POAM-P has good psychometric properties and identifies in clinical practice the 3 main activity patterns, all of which demonstrated excellent internal consistency (Cronbach's alpha coefficients: $0.88,0.89$, and 0.85 for avoidance, pacing, and overdoing subscales, resp.) [36]. Directions are "People who have pain use different ways to do their daily activities. Think about how you usually do your daily activities." Examples of item content include "I stop what I am doing when my pain starts to get worse" (avoidance), "I go back and forth between working and taking breaks when doing an activity" (pacing), and "When I am doing an activity I don't stop until it is finished" (overdoing). The instrument includes 30 questions (10 for each pattern), each scored from 0 to 4 points, and people have to determine the extent to which the proposal fits them. Each subscale is quoted individually. The highest rating reflects a favorite, but not exclusive, behavioral pattern (range: 0-40 points). Without a validated pattern classification methodology, each subscale is handled with continuous scores according to previous research $[5,22,37,38]$.

\subsection{Outcomes}

2.6.1. FCE. The FCE helps determine the individual's safe functional capacity, a very important perspective for people with chronic pain who may be subject to fear of reinjury. Four of the FCE tests with high reliability were conducted during the rehabilitation program, according to Workwell-Isernhagen FCE standards [39, 40]: the floor-to-waist, the waist-to-overhead, the horizontal lifts, and the dominant-handed carrying test. FCE were performed by FCE certified physical therapists who had no knowledge of the POAM-P score and differed from the attending therapist to reduce the risk of bias. During the FCE load-carrying tests, the FCE assessor determines patient's maximum capacity after around five load increases of 2.5 or $5 \mathrm{~kg}$ for each increment. As already detailed [15], to validate a step, the load must be lifted five times for the floor-to-waist, the waist-tooverhead tests, or horizontal lift and carried over a distance of $15 \mathrm{~m}$ for the dominant-handed carrying test. Each step must be performed within 90 seconds, before the assessor increases the weight. Maximal safe performance is determined by the FCE assessor and is based on biomechanical and physiological signs in response to the effort: bulging of prime movers and accessory muscles, very wide base, counterbalance, increase in heart and respiration rate, very slow pace, and safe lifting but inability to maintain control with the addition of any more weight [41]. Unless maximal performance is reached, the test may be interrupted for safety reasons by the assessor or by the patients themselves if they feel unable to perform the task [15].

2.7. Data Collection. To minimize the measurement bias, questionnaires and clinical and demographic data were collected during the 2 days following admission, patients being planned for two 30-minuteperiods devoted to questionnaires. The use of a digital pen allowed data capture and transfer from paper to the data files. FCEs were performed 3 to 4 weeks after admission and were done under the supervision of certified physical therapists, familiar with the tests through regular training and clear instructions.

2.8. Statistical Analyses. Descriptive statistics were expressed as mean and standard deviation for continuous variables, whereas median and interquartile range were used if the distribution was skewed after visual inspection of data. Categorical variables were expressed as count and percentage.

We compared the POAM-P scores according to sociodemographic variables with analysis of variance or Student's $t$-test for binary variables. Eta-squared coefficient was expressed to determine and compare each categorical effect. Following Cohen [42], eta-squared effect size cutoffs were used: 0.01 for small, 0.06 for medium, 0.14 for large effect.

We looked for Pearson's or Spearman's correlations between the POAM-P scores and other variables, following the distribution of variables. Evans's [43] strength of correlations was used: less than 0.20 for very weak, 0.20 to 0.39 
for weak, 0.40 to 0.59 for moderate, and 0.60 or greater for a strong correlation.

To study the association between activity patterns and the FCE tests, we used continuous scores of each of the three subscales of the POAM-P $[5,22,37,38]$. The four FCE tests selected were not available for all patients. A total of 304 patients underwent the floor-to-waist lift, 303 performed the waist-to-overhead lift, 271 performed the horizontal lift, and 298 performed the dominant-handed carrying test. Standard multiple linear regression models were applied to measure the associations between FCE tests and activity patterns (POAM-P subscales) while adjusting these associations for potential confounders. Age, gender, BMI, pain severity, trauma severity, localization of injury, and education were chosen for their potential influence on outcome and activity patterns. The available sample size allowed the chosen parameters in the regression models to keep a minimum of 15 observations per parameter [44]. Only patients with complete data were included in the analysis. As patients did not have to perform every lifting test, the number of cases with complete data may differ for each analysis. To compare effects of variables on the outcome, standardized coefficients are presented [45]. The significance level was set as a probability less than 0.05 . All analyses were performed using Stata 15.1 (StataCorp, College Station, Texas, USA).

\section{Results}

3.1. Sample Characteristics. A total of 304 patients were included. Patients were predominantly middle-aged men (mean age, 42.3 years; $94.7 \%$ men), nearly half were not French native speakers, and $42 \%$ had a low level of education. The median duration between trauma and rehabilitation was 15 months (interquartile range, 10-23 months). Injury locations were $44.2 \%$ upper limb, 42.2\% lower limb, $11.5 \%$ spine injury, and multiple sites for the rest. Most injuries occurred at work (54.3\%) and were classified as minor or moderate according to the Abbreviated Injury Score. The mean pain severity assessed by BPI was $4.03 \pm 1.76$, and the mean pain interference with daily activity was $4.1 \pm 1.97$. The HADS score was just below $7 \pm 4.05$ points for depressive symptoms and $9 \pm 3.98$ points for anxiety symptoms. When looking at perceived ability to perform working tasks assessed with the SFS/HFS, most patients felt they could perform light work. Table 1 details patients' characteristics and their scores for the different psychological questionnaires as well as POAM-P scores for activity patterns. For the entire cohort, the mean maximal performances on the FCE tests were $22.11 \pm 10.38 \mathrm{~kg}$ for the floor-to-waist lift, $13.74 \pm 6.83 \mathrm{~kg}$ for the waist-to-overhead lift, $25.43 \pm 10.38 \mathrm{~kg}$ for the horizontal lift, and $18.70 \pm 8.21 \mathrm{~kg}$ for the dominant-handed carrying test.

Higher avoidance scores were found in nonnative French-speaking patients, whereas lower overdoing/persistence scores were found in patients with lower limb trauma. Effect sizes were small-to-medium (Eta-squared) [42] (Table 2).

Correlations between POAM-P subscales and other variables are presented in Table 3 . The avoidance score was moderately correlated with pacing $(r=0.5)$, whereas only weak correlations were found for pacing and overdoing subscales with other variables (Table 3 ).

\subsection{Relationship between Patterns and Observational Func-} tional Outcomes. Results of the multivariable model analysis are presented in Table 4. The overdoing/persistence pattern was found to have a significant influence on the 4 FCE tests, after adjustment for confounding variables. For the floor-towaist lift, a high overdoing/persistence score was associated with higher performances (coefficient $=0.20 ; p=0.001$ ), meaning that for each supplementary point at the overdoing/persistence score (scale from 0 to 40 points), an increase of $0.20 \mathrm{~kg}$ is expected for the maximal lifted weight. In other words, a difference of $2 \mathrm{~kg}$ at the maximal lifted weight is expected between patients having a difference of 10 points on the overdoing/persistence score. When looking at the standardized coefficient, $3.28 \mathrm{~kg}$ represents the increase expected for the maximal floor-to-waist lift for a gain of 2 standard deviations (16.42 points) at the overdoing/persistence score. We found no influence of avoidance and pacing patterns.

For the waist-to-overhead lift, similar associations were found. A high overdoing/persistence score was associated with higher performances (coefficient $=0.13 ; p=0.004$ ), meaning that for each supplementary point at the score, there will be an increase of $0.13 \mathrm{~kg}$. When looking at the standardized coefficient, an increase of $2.08 \mathrm{~kg}$ is expected every 16.42 points. We found no influence of avoidance and pacing patterns.

For the horizontal lift, a higher overdoing/persistence score was also associated with higher performance (coefficient $=0.31 ; p<0.001$ ), meaning that for each supplementary point on the 40 -point scale, an increase of $0.31 \mathrm{~kg}$ is expected. When looking at the standardized coefficient, an increase of $5.15 \mathrm{~kg}$ is expected every 16.42 points (2 SD) for the maximal horizontal lift.

For the dominant-handed carrying test, overdoing/ persistence was again positively associated with performance (coefficient $=0.19 ; p=0.001$ ) as opposed to pacing, which was negatively associated (coefficient $=-0.14 ; p=0.034$ ). A difference of 1 point on each questionnaire, respectively, represents an increase of $0.19 \mathrm{~kg}$ or a loss of $0.14 \mathrm{~kg}$ on the maximal carrying test. We found no influence of avoidance.

\section{Discussion}

Activity patterns have an influence on physical performance. As expected, our hypothesis regarding overdoing/persistence was verified, as it was positively associated with the 4 FCE tests. Pacing was found to be negatively associated only with carrying with the dominant-hand, but no association was found for the 3 other tests, whereas avoidance was not found to be associated with any of the 4 FCE tests.

Overdoing/persistence was associated with the 4 FCE tests studied. An increase of 10 points on the overdoing/ persistence score represents an increase ranging from $1.3 \mathrm{~kg}$ for maximal waist-to-overhead lift to $3.1 \mathrm{~kg}$ for maximal 
TABLE 1: Summary statistics.

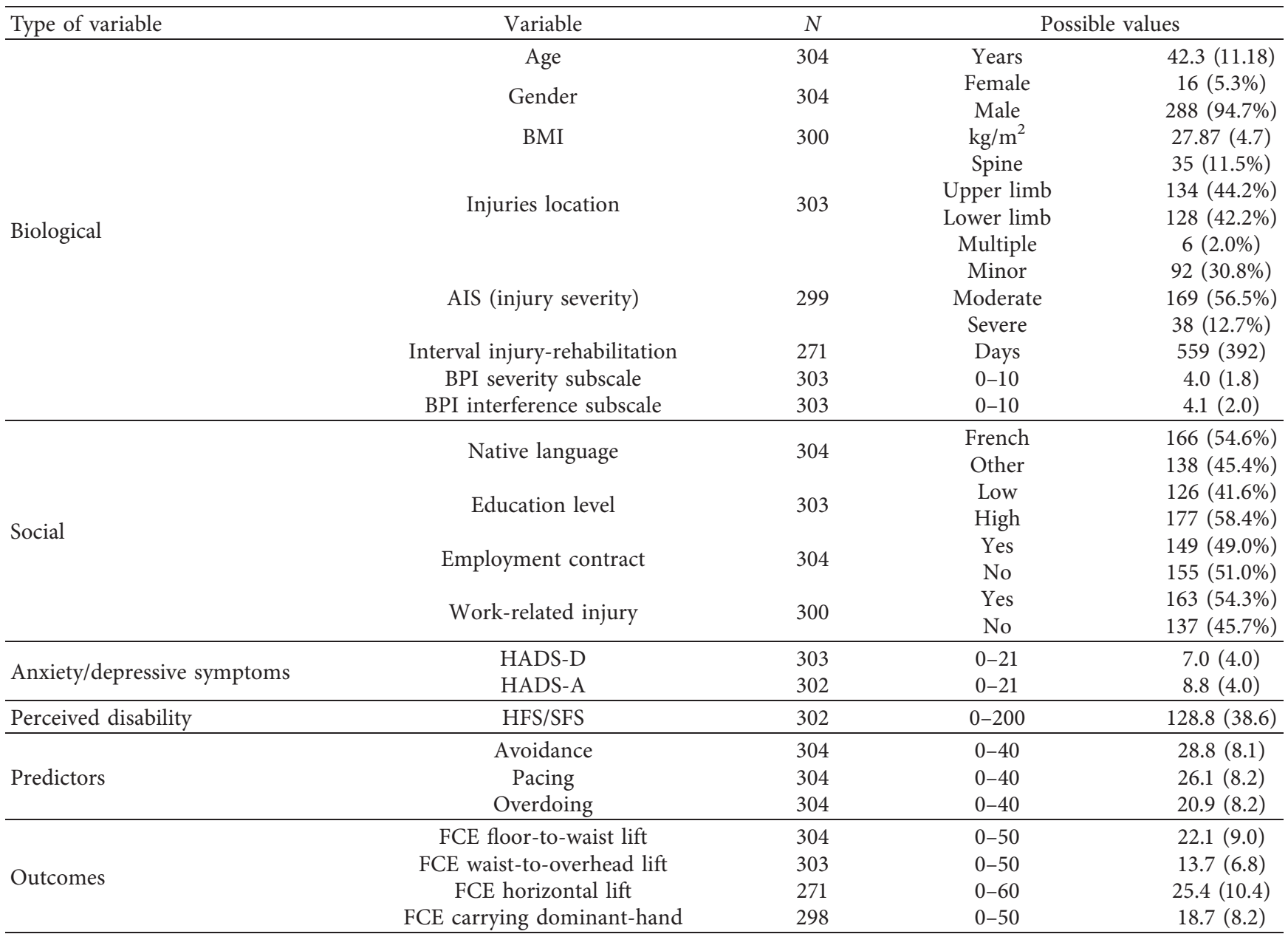

$N=$ available data for each variable; possible values = range for continuous variables and categories for dichotomized variables; descriptive statistics = mean value and standard deviation for continuous variables and absolute number and relative number for binary variables, age in years. BMI $=$ body mass index $(\mathrm{kg} /$ $\mathrm{m}^{2}$ ); AIS = Abbreviated Injury Scale, interval between injury and hospitalization in days; BPI = Brief Pain Inventory; HADS-D = Hospital Anxiety and Depression Scale-depression subscale; HADS-A = Hospital Anxiety and Depression Scale-anxiety subscale; HFS = Hand Function Sort and SFS = Spinal Function Sort, normalized to 200 points; FCE = Functional Capacity Evaluation (kg).

horizontal lift. As weights have to be carried 5 times to validate each step for the lifting tests, the differences for the total manipulated weights are 5 times heavier: $6.5 \mathrm{~kg}$ for the maximal waist-to-overhead lift to $15 \mathrm{~kg}$ for the maximal horizontal lift for an increase of 10 points on the overdoing/ persistence score. When looking at the standardized coefficient that allows comparison of numeric and binary variables, the expected gain for an increase of 16 points at the overdoing/persistence score (2 SD) ranged from $2.1 \mathrm{~kg}$ for the maximal waist-to-overhead lift to $5.1 \mathrm{~kg}$ for the maximal horizontal lift. These gains counterbalance the expected loss of performance associated with age, as the expected change for age ranged from a $1.9 \mathrm{~kg}$ loss for the carrying with dominant-hand test to $3.4 \mathrm{~kg}$ for the floor-to-waist lift, for every increase of 22 years ( $2 \mathrm{SD}$ ). If we compare the effect with gender, it also partially compensates for the influence of female gender on physical performances during FCE.

The influence of the overdoing/persistence pattern is debated in the literature, which is mainly based on subjective measures: Cane et al. found a positive association with disability in a population of patients with chronic pain recruited from a pain clinic, with a median pain duration of 7 years, and a high proportion of fibromyalgia, in contrast to our population [5], whereas others found mixed associations of task persistence $[6,8]$, or no association [47]. The association of overdoing/persistence with better functioning seems to be in accordance to those of Hasenbring, who found a lower perceived disability in a subgroup of patients called "eustress-endurant" [48], and with Luthi who found positive associations of overdoing/persistence with functioning [22]. Interestingly in a recent publication, Cane found that overdoing patients had a higher perceived functioning, assessed with the Pain Disability Index compared with patients with high avoidance and pacing subscales [3]. This difference with a previous study [5] is not discussed by Cane but may be linked with the fact that patients were grouped in clusters in the latter study, whereas an analysis of each subscale was done in the former. An association of overdoing/persistence patterns of activity with observational measures was found in few studies: Huijnen 
TABLE 2: Mean POAM-P scores according to sociodemographic variables.

\begin{tabular}{|c|c|c|c|c|c|c|c|c|c|c|c|}
\hline \multirow{2}{*}{ Variable } & \multirow{2}{*}{$N$} & \multirow{2}{*}{ Possible values } & \multicolumn{3}{|c|}{ Avoidance } & \multicolumn{3}{|c|}{ Pacing } & \multicolumn{3}{|c|}{ Overdoing } \\
\hline & & & Mean & Eta-squared & $p$ value & Mean & Eta-squared & $p$ value & Mean & Eta-squared & $p$ value \\
\hline \multirow{2}{*}{ Gender } & 16 & Female & 27.5 & \multirow{2}{*}{0.0014} & \multirow{2}{*}{0.518} & 28.7 & \multirow{2}{*}{0.006} & \multirow{2}{*}{0.182} & 22.8 & \multirow{2}{*}{0.003} & \multirow{2}{*}{0.342} \\
\hline & 288 & Male & 28.9 & & & 25.9 & & & 20.8 & & \\
\hline \multirow{4}{*}{ Injuries location } & 134 & Upper limb & 28.6 & \multirow{4}{*}{0.014} & \multirow{4}{*}{0.244} & 26.0 & \multirow{4}{*}{0.018} & \multirow{4}{*}{0.143} & 21.1 & \multirow{4}{*}{0.028} & \multirow{4}{*}{0.037} \\
\hline & 128 & Lower limb & 29.5 & & & 26.0 & & & 19.7 & & \\
\hline & 35 & Back & 27.5 & & & 27.7 & & & 23.6 & & \\
\hline & 6 & Multiple sites & 23.8 & & & 19.3 & & & 25.7 & & \\
\hline \multirow{3}{*}{ AIS } & 92 & Minor & 29.6 & \multirow{3}{*}{0.010} & \multirow{3}{*}{0.220} & 26.2 & \multirow{3}{*}{0.000} & \multirow{3}{*}{0.955} & 20.5 & \multirow{3}{*}{0.002} & \multirow{3}{*}{0.786} \\
\hline & 169 & Moderate & 29.1 & & & 26.0 & & & 21.0 & & \\
\hline & 38 & Severe & 26.9 & & & 26.4 & & & 21.5 & & \\
\hline \multirow{2}{*}{ Native language } & 166 & French & 27.5 & \multirow{2}{*}{0.032} & \multirow{2}{*}{0.002} & 25.5 & \multirow{2}{*}{0.007} & \multirow{2}{*}{0.156} & 21.6 & \multirow{2}{*}{0.009} & \multirow{2}{*}{0.098} \\
\hline & 138 & Other & 30.4 & & & 26.8 & & & 20.1 & & \\
\hline Education & 126 & $<9$ years & 29.2 & 0.002 & 0.455 & 26.9 & 0.007 & 0.149 & 20.5 & 0.001 & 0.528 \\
\hline & 177 & $\geq 9$ years & 28.5 & & 0.450 & 25.5 & & 0.149 & 21.2 & 0.001 & 0.320 \\
\hline Employment contract & 149 & Yes & 28.8 & $<0.001$ & 0.962 & 26.5 & 0.003 & 0.353 & 20.7 & $<0001$ & 0688 \\
\hline Emproyment contract & 155 & No & 28.8 & $<0.001$ & 0.902 & 25.6 & 0.003 & 0.353 & 21.1 & $<0.001$ & 0.080 \\
\hline Work-related injury & 163 & Yes & 28.4 & 003 & 307 & 26.1 & & & 20.3 & & \\
\hline work-rerated mjury & 137 & No & 29.3 & 0.003 & 0.307 & 26.0 & $<0.001$ & 0.890 & 21.7 & 0.007 & $0.15 /$ \\
\hline
\end{tabular}

Student's $t$-tests or analysis of variance to compare groups. POAM-P: Patterns of Activity Measures-pain; AIS = Abbreviated Injury Scale. Effect size (etasquared) [42]: $0.01=$ small; $0.06=$ medium; $0.14=$ large.

TABLE 3: Correlations between the three POAM-P subscales and other variables.

\begin{tabular}{|c|c|c|c|c|c|c|}
\hline \multirow{2}{*}{ Variable } & \multicolumn{2}{|c|}{ Avoidance } & \multicolumn{2}{|c|}{ Pacing } & \multicolumn{2}{|c|}{ Overdoing } \\
\hline & Correlation & $p$ value & Correlation & $p$ value & Correlation & $p$ value \\
\hline Avoidance & 1.00 & & 0.50 & $<0.001$ & -0.29 & $<0.001$ \\
\hline Pacing & 0.50 & $<0.001$ & 1.00 & & -0.12 & 0.033 \\
\hline Overdoing & -0.29 & $<0.001$ & -0.12 & 0.033 & 1.00 & \\
\hline BPI-interference & 0.31 & $<0.001$ & 0.05 & 0.427 & -0.16 & 0.004 \\
\hline BPI-severity & 0.28 & $<0.001$ & 0.07 & 0.253 & -0.17 & 0.002 \\
\hline HADS-D & 0.25 & $<0.001$ & 0.01 & 0.866 & -0.14 & 0.012 \\
\hline HADS-A & 0.13 & 0.052 & -0.05 & 0.352 & -0.05 & 0.402 \\
\hline HFS/SFS & -0.23 & $<0.001$ & -0.15 & 0.007 & 0.23 & $<0.001$ \\
\hline Age & -0.01 & 0.851 & 0.27 & $<0.001$ & -0.07 & 0.253 \\
\hline FCE low lift & -0.11 & 0.121 & -0.18 & 0.001 & 0.21 & $<0.001$ \\
\hline FCE horizontal lift & -0.09 & 0.209 & -0.21 & $<0.001$ & 0.27 & $<0.001$ \\
\hline FCE high lift & -0.06 & 0.384 & -0.15 & 0.008 & 0.16 & 0.007 \\
\hline FCE carry dom. hand & -0.06 & 0.374 & -0.17 & 0.004 & 0.22 & $<0.001$ \\
\hline
\end{tabular}

BPI = Brief Pain Inventory; HADS-D = Hospital Anxiety and Depression Scale-depression subscale; HADS-A = Hospital Anxiety and Depression Scaleanxiety subscale; HFS = Hand Function Sort and SFS = Spinal Function Sort, normalized to 200 points; FCE = Functional Capacity Evaluation $(\mathrm{kg})$. Correlation coefficient [46]: 0.20 to $0.39=$ weak; 0.4 to $0.59=$ moderate; 0.60 and greater $=$ strong.

et al. could not find an association of patterns with daily life activity measured with accelerometers in patients with chronic low-back pain [12], whereas Andrews et al. found an association between perceived overactivity and physical activity measured with accelerometers [49]. These contradictory results may be explained by the different settings and populations of patients of the studies and the various strategies that may be used depending on the context $[4,50]$. In the present study patients were asked to give their maximal safe effort. High demand instructions have been shown to improve reliability of tests [51] and even if instructions to patients may influence strength measurements [51-53], the specific influence of a demand on a preferential activity pattern is not known. We suppose high demand instructions improve performance of every patient and we do not think it would annihilate pacing or avoidance patterns, as we found similar associations in a former study addressing physical fitness tests without the demand to give a maximum effort [22].

We found no significant association of pacing with the 3 FCE lifting tests. Other studies using the POAM-P and addressing subjective measures of functioning showed contradictory results: Cane et al. found lower perceived disability for patients with a high level of pacing [5], whereas Kindermans found pacing to be positively correlated with disability [6]. The negative association with maximal carrying with dominant-hand performance may be in accordance with results found in another study in the same 
TABLE 4: Multiple regression models for FCE performances.

\begin{tabular}{|c|c|c|c|c|c|c|c|}
\hline Outcome & Variable & $n$ & Adj. $r^{2}$ & Coeff. & CI $(95 \%)$ & $p$ & Std. coeff. \\
\hline \multirow{3}{*}{ FCE floor-to-waist lift } & Avoidance & \multirow{3}{*}{293} & \multirow{3}{*}{0.25} & -0.00 & $-0.14 ; 0.14$ & 0.984 & -0.02 \\
\hline & Pacing & & & -0.07 & $-0.21 ; 0.06$ & 0.278 & -1.22 \\
\hline & Overdoing & & & 0.20 & $0.08 ; 0.32$ & 0.001 & 3.28 \\
\hline \multirow{3}{*}{ FCE waist-to-overhead lift } & Avoidance & \multirow{3}{*}{292} & \multirow{3}{*}{0.31} & 0.06 & $-0.04 ; 0.16$ & 0.211 & 1.03 \\
\hline & Pacing & & & -0.07 & $-0.16 ; 0.03$ & 0.184 & -1.09 \\
\hline & Overdoing & & & 0.13 & $0.04 ; 0.21$ & 0.004 & 2.08 \\
\hline \multirow{3}{*}{ FCE horizontal lift } & Avoidance & \multirow{3}{*}{260} & \multirow{3}{*}{0.24} & 0.08 & $-0.09 ; 0.26$ & 0.358 & 1.33 \\
\hline & Pacing & & & -0.15 & $-0.31 ; 0.02$ & 0.083 & -2.43 \\
\hline & Overdoing & & & 0.31 & $0.16 ; 0.47$ & $<.001$ & 5.15 \\
\hline \multirow{3}{*}{ FCE carrying dominant-hand } & Avoidance & \multirow{3}{*}{288} & \multirow{3}{*}{0.16} & 0.09 & $-0.04 ; 0.22$ & 0.173 & 1.49 \\
\hline & Pacing & & & -0.14 & $-0.27 ;-0.01$ & 0.034 & -2.32 \\
\hline & Overdoing & & & 0.19 & $0.07 ; 0.30$ & 0.001 & 3.11 \\
\hline
\end{tabular}

Adjusted with confounding variables: age, body mass index (BMI), gender, trauma location, trauma severity, pain, and education. Std. coeff.: standardized coefficient for a change of $2 \mathrm{SD}$.

setting, in which high pacing was associated with lower walking performances [22]. This association with lower physical performance may be consistent with other studies, which found pacing to be associated with disability [4] or lower level of physical activity [54]. We have no definite explanation about the fact that pacing was found to be negatively associated with only one of the four tests. Our hypothesis is that behavioral strategies vary according to the context [4]. When looking at the 4 studied FCE tests, the dominant-hand carrying test may indeed represent a more complex activity compared to the other 3 tests. It requires not only lifting a weight from one point to another in incremental stages every 5 succeeded lifts but also walking for $15 \mathrm{~m}$ while carrying a weight that will increase with every succeeding stage. Moreover, the test itself takes longer than the 3 lifting tests, and pain-contingent pacing addressed with the POAM-P might become preferential in this situation, as opposed to other lifting tasks that may not promote it.

We found no association between the avoidance pattern and any of the 4 FCE tests. These results may seem surprising at first glance, as this pattern is associated with poor functioning in the literature $[7,15,47,55]$. However, the few studies that also used observational measures of physical activity showed that there was more often no significant influence $[10,12,22]$. This discrepancy between subjective perception and observation can have a major influence/ impact on the management of these patients. Indeed, successfully challenging these perceptions by comparing them with the actual performance achieved during a rehabilitation program, for instance, could be of great help in the care process. For some patients, this could lead to a more realistic interpretation of their own situation, especially among those who favor avoidance or pain-contingent pacing strategies $[3,22]$.

4.1. Implications of This Study. Our results highlight the importance of using observational measures in studies addressing the influence of behavioral patterns in patients with chronic pain. Such measures seem important, as subjective tools cannot just replace them and may lead to inaccurate or incomplete conclusions [56]. These observational measures are moreover not so costly and time-consuming and can be easily integrated into a therapeutic program often performed by physiotherapists or occupational therapists for their functional component [56].

There is a positive association of overdoing/persistence pattern with objective physical activity measurements. Even if we cannot draw causal interpretation, our results suggest a behavioral strategy based on task-contingent persistence instructions may be more effective than modulation strategies often proposed in the rehabilitation of chronic pain [7]. Without asking patients to exert themselves beyond their limits (excessive persistence), the positive associations of task-contingence persistence with physical performance should promote strategies that enable choosing an activity, precisely determining the level of effort at which it can be safely performed, and setting the training conditions that will allow the patient to complete this task, without pain or fatigue being grounds for stopping the activity. It would be interesting to evaluate whether this approach makes it possible to break the association between pain, fatigue, and disability that often remains observed, including therapeutic interventions based on activity modulation [57].

4.2. Strengths and Limitations. This is the first study addressing measures of activity patterns in the context of patients with chronic musculoskeletal pain after trauma. The use of FCE as an observational measure is another strength because of its reliability and ability to determine a maximum safe effort for the patient. However, this study also has some limitations. First, the sample mainly consisted of men addressed for specialized rehabilitation. The setting in itself may influence activity patterns and indeed may limit the generalization of our results. This type of study should therefore be repeated in samples that include a greater number of women. This would be all the more interesting as the overdoing/persistence pattern has been positively associated with the female gender [5]. A second limitation may be the interval between activity pattern evaluation with the POAM-P and FCE tests, as activity patterns may change 
during the rehabilitation program [3]. However, the associations found indicate that the activity patterns predict future behavior. Another limitation is related to the POAM$\mathrm{P}$ questionnaire and the interpretation of overdoing/persistence. For Kindermans et al. [6], the POAM-P, as compared with other questionnaires that can be used, is thought to reflect task-contingent persistence more than excessive persistence or pain-contingent persistence, which may have a worse outcome. Finally, this observational study and the type of analysis conducted allows us to state an influence of some activity patterns on FCE performances but does not enable us to establish a cause-effect relationship.

\section{Conclusion}

Our results suggest that the overdoing/persistence pattern as defined in the POAM-P is positively associated with the following functional tasks: carrying a load from the ground to the waist, carrying a load from the waist to the head, lifting a load horizontally, and moving a load with the dominant hand, all of which are common tasks in daily and professional life. Further studies will be needed to determine whether there is a causal relationship between this pattern and physical performance.

\section{Data Availability}

The data used to support the findings of this study are available from the corresponding author upon request.

\section{Conflicts of Interest}

The authors declare that there are no conflicts of interest regarding the publication of this paper.

\section{Acknowledgments}

The authors thank the nursing team who collected the questionnaires and the physiotherapists who performed FCE. The authors are also grateful to Caroline Praz and the research assistants at the Institute for Research in Rehabilitation, Clinique Romande de Réadaptation, Sion, Switzerland, for their support with data acquisition. This research was supported by a donation of the Loterie Suisse Romande (LORO).

\section{References}

[1] F. J. Keefe and J. Dunsmoret, "Pain behavior concepts and controversies," APS Journal, vol. 1, no. 2, pp. 92-100, 1992.

[2] B. N. Rosenbloom, S. Khan, C. McCartney, and J. Katz, "Systematic review of persistent pain and psychological outcomes following traumatic musculoskeletal injury," Journal of Pain Research, vol. 6, pp. 39-51, 2013.

[3] D. Cane, W. R. Nielson, and D. Mazmanian, "Patterns of painrelated activity: replicability, treatment-related changes, and relationship to functioning," Pain, vol. 159, no. 12, pp. 2522-2529, 2018.

[4] L. M. McCracken and V. M. Samuel, "The role of avoidance, pacing, and other activity patterns in chronic pain," Pain, vol. 130, no. 1-2, pp. 119-125, 2007.
[5] D. Cane, W. R. Nielson, M. McCarthy, and D. Mazmanian, "Pain-related activity patterns: measurement, interrelationships, and associations with psychosocial functioning," The Clinical Journal of Pain, vol. 29, no. 5, pp. 435-442, 2013.

[6] H. P. Kindermans, J. Roelofs, M. E. Goossens, I. P. Huijnen, J. A. Verbunt, and J. W. S. Vlaeyen, "Activity patterns in chronic pain: underlying dimensions and associations with disability and depressed mood," The Journal of Pain, vol. 12, no. 10, pp. 1049-1058, 2011.

[7] N. E. Andrews, J. Strong, and P. J. Meredith, "Activity pacing, avoidance, endurance, and associations with patient functioning in chronic pain: a systematic review and meta-analysis," Archives of Physical Medicine and Rehabilitation, vol. 93, no. 11, pp. 2109-2121, 2012.

[8] M. A. Ferreira-Valente, J. L. Pais-Ribeiro, and M. P. Jensen, "Associations between psychosocial factors and pain intensity, physical functioning, and psychological functioning in patients with chronic pain: a cross-cultural comparison," The Clinical Journal of Pain, vol. 30, no. 8, pp. 713-723, 2014.

[9] W. R. Nielson, M. P. Jensen, P. A. Karsdorp, and J. W. Vlaeyen, "Activity pacing in chronic pain: concepts, evidence, and future directions," The Clinical Journal of Pain, vol. 29, no. 5, pp. 461-468, 2013.

[10] E. Fehrmann, K. Tuechler, T. Kienbacher et al., "Comparisons in muscle function and training rehabilitation outcomes between avoidance-endurance model subgroups," The Clinical Journal of Pain, vol. 33, no. 10, pp. 912-920, 2017.

[11] M. G. van Weering, M. M. Vollenbroek-Hutten, and H. J. Hermens, "The relationship between objectively and subjectively measured activity levels in people with chronic low back pain," Clinical Rehabilitation, vol. 25, no. 3, pp. 256-263, 2011.

[12] I. P. Huijnen, J. A. Verbunt, M. L. Peters et al., "Differences in activity-related behaviour among patients with chronic low back pain," European Journal of Pain, vol. 15, no. 7, pp. 748-755, 2011.

[13] T. Pincus, R. J. Smeets, M. J. Simmonds, and M. J. L. Sullivan, "The fear avoidance model disentangled: improving the clinical utility of the fear avoidance model," The Clinical Journal of Pain, vol. 26, no. 9, pp. 739-746, 2010.

[14] P. Oesch, K. Meyer, S. Bachmann, K. B. Hagen, and N. K. Vollestad, "Comparison of two methods for interpreting lifting performance during functional capacity evaluation," Physical Therapy, vol. 92, no. 9, pp. 1130-1140, 2012.

[15] J. Tuscher, C. Burrus, P. Vuistiner, B. Leger, G. Rivier, and F. Luthi, "Predictive value of the fear-avoidance model on functional capacity evaluation," Journal of Occupational Rehabilitation, vol. 28, no. 3, pp. 513-522, 2018.

[16] S. Brouwer, M. F. Reneman, P. U. Dijkstra, J. W. Groothoff, J. M. Schellekens, and L. N. Goeken, "Test-retest reliability of the isernhagen work systems functional capacity evaluation in patients with chronic low back pain," Journal of Occupational Rehabilitation, vol. 13, no. 4, pp. 207-218, 2003.

[17] D. P. Gross and M. C. Battie, "Reliability of safe maximum lifting determinations of a functional capacity evaluation," Physical Therapy, vol. 82, no. 4, pp. 364-371, 2002.

[18] S. J. Isernhagen, D. L. Hart, and L. M. Matheson, "Reliability of independent observer judgments of level of lift effort in a kinesiophysical functional capacity evaluation," Work, vol. 12, no. 2, pp. 145-150, 1999.

[19] D. P. Gross and M. C. Battie, "Factors influencing results of functional capacity evaluations in workers' compensation claimants with low back pain," Physical Therapy, vol. 85, no. 4, pp. 315-322, 2005. 
[20] J. M. Lackner and A. M. Carosella, "The relative influence of perceived pain control, anxiety, and functional self efficacy on spinal function among patients with chronic low back pain," Spine, vol. 24, no. 21, pp. 2254-2260, 1999.

[21] R. van Abbema, S. E. Lakke, M. F. Reneman et al., "Factors associated with functional capacity test results in patients with non-specific chronic low back pain: a systematic review," Journal of Occupational Rehabilitation, vol. 21, no. 4, pp. 455-473, 2011.

[22] F. Luthi, P. Vuistiner, C. Favre, R. Hilfiker, and B. Leger, "Avoidance, pacing, or persistence in multidisciplinary functional rehabilitation for chronic musculoskeletal pain: an observational study with cross-sectional and longitudinal analyses," PLoS One, vol. 13, no. 9, Article ID e0203329, 2018.

[23] L. Karlsson, B. Gerdle, E. P. Takala, G. Andersson, and B. Larsson, "Experiences and attitudes about physical activity and exercise in patients with chronic pain: a qualitative interview study," Journal of Pain Research, vol. 11, pp. 133-144, 2018.

[24] S. J. Kamper, A. T. Apeldoorn, A. Chiarotto et al., "Multidisciplinary biopsychosocial rehabilitation for chronic low back pain: cochrane systematic review and meta-analysis," $B M J$, vol. 350, no. 5, p. h444, 2015.

[25] S. P. Baker, B. O’Neill, W. Haddon Jr., and W. B. Long, "The injury severity score: a method for describing patients with multiple injuries and evaluating emergency care," The Journal of Trauma: Injury, Infection, and Critical Care, vol. 14, no. 3, pp. 187-196, 1974.

[26] A. S. Zigmond and R. P. Snaith, "The hospital anxiety and depression scale," Acta Psychiatrica Scandinavica, vol. 67, no. 6, pp. 361-370, 1983.

[27] M. M. Veehof, P. M. Ten Klooster, E. Taal, G. J. Westerhof, and E. T. Bohlmeijer, "Psychometric properties of the Dutch five facet mindfulness questionnaire (FFMQ) in patients with fibromyalgia," Clinical Rheumatology, vol. 30, no. 8, pp. 1045-1054, 2011.

[28] D. Razavi, N. Delvaux, C. Farvacques, and E. Robaye, "Validation de la version française du HADS dans une population de patients cancéreux hospitalisés. [Validation of the French version of the hospital anxiety and depression scale (HADS) in a population of hospitalized cancer patients]," Revue de Psychologie Appliquée, vol. 39, no. 4, pp. 295-307, 1989.

[29] C. S. Cleeland and K. M. Ryan, "Pain assessment: global use of the brief pain inventory," Annals of the Academy of Medicine Singapore, vol. 23, no. 2, pp. 129-138, 1994.

[30] J. Poundja, D. Fikretoglu, S. Guay, and A. Brunet, "Validation of the French version of the brief pain inventory in Canadian veterans suffering from traumatic stress," Journal of Pain and Symptom Management, vol. 33, no. 6, pp. 720-726, 2007.

[31] G. Tan, M. P. Jensen, J. I. Thornby, and B. F. Shanti, "Validation of the brief pain inventory for chronic nonmalignant pain," The Journal of Pain, vol. 5, no. 2, pp. 133-137, 2004.

[32] M. Konzelmann, C. Burrus, R. Hilfiker, G. Rivier, O. Deriaz, and F. Luthi, "Cross-cultural adaptation, reliability, internal consistency and validation of the hand function sort (HFS(c)) for French speaking patients with upper limb complaints," Journal of Occupational Rehabilitation, vol. 25, no. 1, pp. 18-24, 2015.

[33] L. N. Matheson, V. K. Kaskutas, and D. Mada, "Development and construct validation of the hand function sort," Journal of Occupational Rehabilitation, vol. 11, no. 2, pp. 75-86, 2001.

[34] S. Borloz, M. A. Trippolini, P. Ballabeni, F. Luthi, and O. Deriaz, "Cross-cultural adaptation, reliability, internal consistency and validation of the spinal function sort (SFS) for
French- and German-speaking patients with back complaints," Journal of Occupational Rehabilitation, vol. 22, no. 3, pp. 387-393, 2012.

[35] L. N. Matheson, M. L. Matheson, and J. Grant, "Development of a measure of perceived functional ability," Journal of Occupational Rehabilitation, vol. 3, no. 1, pp. 15-30, 1993.

[36] C. Benaim, B. Leger, P. Vuistiner, and F. Luthi, "Validation of the French version of the "patterns of activity measure" in patients with chronic musculoskeletal pain," Pain Research and Management, vol. 2017, Article ID 6570394, 7 pages, 2017.

[37] D. Cane, M. McCarthy, and D. Mazmanian, "Obstacles to activity pacing: assessment, relationship to activity and functioning," Pain, vol. 157, no. 7, pp. 1508-1514, 2016.

[38] M. Racine, E. Castarlenas, R. de la Vega et al., "Sex differences in psychological response to pain in patients with fibromyalgia syndrome," The Clinical Journal of Pain, vol. 31, no. 5, pp. 425-432, 2015.

[39] S. J. Isernhagen, "Functional capacity evaluation: rationale, procedure, utility of the kinesiophysical approach," Journal of Occupational Rehabilitation, vol. 2, no. 3, pp. 157-168, 1992.

[40] S. Bieniek and M. Bethge, "The reliability of WorkWell systems functional capacity evaluation: a systematic review," BMC Musculoskeletal Disorders, vol. 15, no. 1, p. 106, 2014.

[41] M. F. Reneman, A. S. Fokkens, P. U. Dijkstra, J. H. Geertzen, and J. W. Groothoff, "Testing lifting capacity: validity of determining effort level by means of observation," Spine, vol. 30, no. 2, pp. E40-E46, 2005.

[42] J. Cohen, Statistical Power Analysis for the Behavioral Sciences, Lawrence Erlbaum Associates, New York, NY, USA, 2nd edition, 1988.

[43] J. D. Evans, Straightforward Statistics for the Behavioral Sciences, Thomson Brooks/Cole Publishing, Belmont, CA, USA, 1996.

[44] F. Harrell, Regression Modeling Strategies: With Applications to Linear Models, Logistic and Ordinal Regression, and Survival Analysis, Springer, Berlin, Germany, 2015.

[45] A. Gelman, "Scaling regression inputs by dividing by two standard deviations," Statistics in Medicine, vol. 27, no. 15, pp. 2865-2873, 2008.

[46] J. D. Evans, Straightforward Statistics for the Behavioral Sciences, Brooks/Cole Pub. Co., Pacific Grove, CA, USA, 1996.

[47] M. Racine, S. Galan, R. de la Vega et al., "Pain-related activity management patterns and function in patients with fibromyalgia syndrome," The Clinical Journal of Pain, vol. 34, no. 2, pp. 122-129, 2018.

[48] M. I. Hasenbring, D. Hallner, B. Klasen, I. Streitlein-Bohme, R. Willburger, and H. Rusche, "Pain-related avoidance versus endurance in primary care patients with subacute back pain: psychological characteristics and outcome at a 6-month follow-up," Pain, vol. 153, no. 1, pp. 211-217, 2012.

[49] N. E. Andrews, J. Strong, and P. J. Meredith, "Overactivity in chronic pain: is it a valid construct?" Pain, vol. 156, no. 10, pp. 1991-2000, 2015.

[50] D. Antcliff, M. Campbell, S. Woby, and P. Keeley, "Activity pacing is associated with better and worse symptoms for patients with long-term conditions," The Clinical Journal of Pain, vol. 33, no. 3, pp. 205-214, 2016.

[51] L. Matheson, V. Mooney, V. Caiozzo et al., "Effect of instructions on isokinetic trunk strength testing variability, reliability, absolute value, and predictive validity," Spine, vol. 17, no. 8, pp. 914-921, 1992.

[52] B. N. Leitzelar, S. Razon, U. Tokac, S. Dieringer, C. Book, and L. W. Judge, "Effects of a supportive audience on a handgrip squeezing task in adults," International Journal of Exercise Science, vol. 9, no. 1, pp. 4-15, 2017. 
[53] A. O'Sullivan and K. O'Sullivan, “The effect of combined visual feedback and verbal encouragement on isokinetic concentric performance in healthy females," Isokinetics \& Exercise Science, vol. 16, no. 1, pp. 47-53, 2008.

[54] S. L. Murphy, D. M. Smith, and N. B. Alexander, "Measuring activity pacing in women with lower-extremity osteoarthritis: a pilot study," American Journal of Occupational Therapy, vol. 62, no. 3, pp. 329-334, 2008.

[55] E. L. Zale, K. L. Lange, S. A. Fields, and J. W. Ditre, "The relation between pain-related fear and disability: a metaanalysis," The Journal of Pain, vol. 14, no. 10, pp. 1019-1030, 2013.

[56] B. J. Guildford, C. M. Jacobs, A. Daly-Eichenhardt, W. Scott, and L. M. McCracken, "Assessing physical functioning on pain management programmes: the unique contribution of directly assessed physical performance measures and their relationship to self-reports," British Journal of Pain, vol. 11, no. 1, pp. 46-57, 2017.

[57] S. L. Murphy and A. L. Kratz, "Activity pacing in daily life: a within-day analysis," Pain, vol. 155, no. 12, pp. 2630-2637, 2014. 